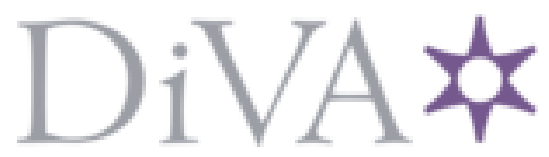

http://www.diva-portal.org

This is the published version of a paper published in Review of Scientific Instruments.

Citation for the original published paper (version of record):

Plogmaker, S., Linusson, P., Eland, J H., Baker, N., Johansson, E M. et al. (2012)

Versatile high-repetition-rate phase-locked chopper system for fast timing experiments in the vacuum ultraviolet and $\mathrm{x}$-ray spectral region.

Review of Scientific Instruments, 83(1): 013115

http://dx.doi.org/10.1063/1.3677329

Access to the published version may require subscription.

N.B. When citing this work, cite the original published paper.

Permanent link to this version:

http://urn.kb.se/resolve?urn=urn:nbn:se:uu:diva- 168797 


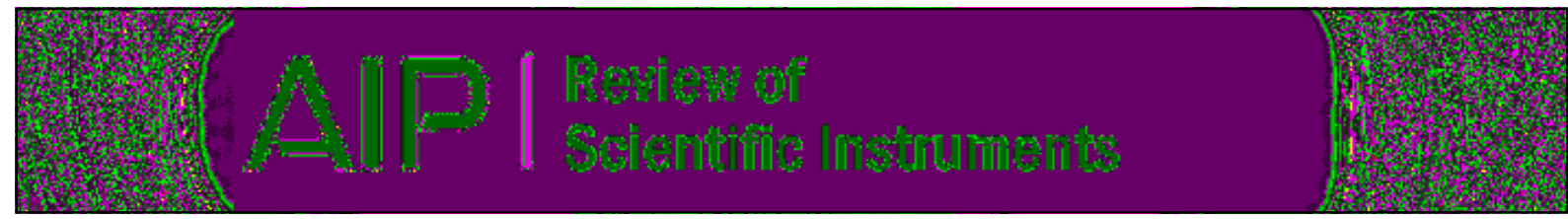

Versatile high-repetition-rate phase-locked chopper system for fast timing experiments in the vacuum ultraviolet and $x$-ray spectral region

Stefan Plogmaker, Per Linusson, John H. D. Eland, Neville Baker, Erik M. J. Johansson, Håkan Rensmo, Raimund Feifel, and Hans Siegbahn

Citation: Review of Scientific Instruments 83, 013115 (2012); doi: 10.1063/1.3677329

View online: http://dx.doi.org/10.1063/1.3677329

View Table of Contents: http://scitation.aip.org/content/aip/journal/rsi/83/1?ver=pdfcov

Published by the AIP Publishing

Articles you may be interested in

High-Precision Time Delay Control with Continuous Phase Shifter for Pump-Probe Experiments Using

Synchrotron Radiation Pulses

AIP Conf. Proc. 1234, 951 (2010); 10.1063/1.3463375

An Extreme Flux Vacuum Ultraviolet/Ultraviolet Beamline For The Measurement Of Biological Circular Dichroism AIP Conf. Proc. 705, 440 (2004); 10.1063/1.1757828

The Russian-German Soft X-Ray Beamline at BESSY II

AIP Conf. Proc. 705, 309 (2004); 10.1063/1.1757795

Characteristics of Relativistic Nonlinear Thomson scattering of an intense laser field as ultrashort x-ray source AIP Conf. Proc. 641, 373 (2002); 10.1063/1.1521046

Direct measurement of the time structure of ultrashort x-ray pulses from a storage ring

AIP Conf. Proc. 521, 479 (2000); 10.1063/1.1291834

\section{Ф SHIMADZU Powerful, Multi-functional UV-Vis-NIR and Excellence in Science FTIR Spectrophotometers}

Providing the utmost in sensitivity, accuracy and resolution for applications in materials characterization and nano research

- Photovoltaics

- Polymers

- Thin films

- Paints

- Ceramics

- DNA film structures

- Coatings

- Packaging materials
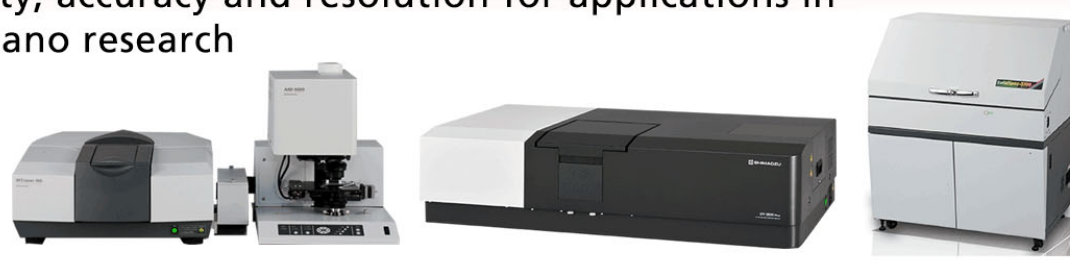


\title{
Versatile high-repetition-rate phase-locked chopper system for fast timing experiments in the vacuum ultraviolet and $x$-ray spectral region
}

\author{
Stefan Plogmaker, ${ }^{1, \text { a) }}$ Per Linusson, ${ }^{2}$ John H. D. Eland, ${ }^{1,3}$ Neville Baker, ${ }^{3}$ Erik M. J. \\ Johansson, ${ }^{1}$ Håkan Rensmo, ${ }^{1, b)}$ Raimund Feifel, ${ }^{1, c)}$ and Hans Siegbahn ${ }^{1}$ \\ ${ }^{1}$ Department of Physics and Astronomy, Uppsala University, Box 516, SE-751 20 Uppsala, Sweden \\ ${ }^{2}$ Department of Physics, Stockholm University, AlbaNova University Center, SE-106 91 Stockholm, Sweden \\ ${ }^{3}$ Department of Chemistry, Physical and Theoretical Chemistry Laboratory, Oxford University, South Parks \\ Road, Oxford OX1 3QZ, United Kingdom
}

(Received 21 September 2011; accepted 21 December 2011; published online 27 January 2012)

\begin{abstract}
A novel light chopper system for fast timing experiments in the vacuum-ultraviolet (VUV) and $\mathrm{X}$-ray spectral region has been developed. It can be phase-locked and synchronized with a synchrotron radiation storage ring, accommodating repetition rates in the range of $\sim 8$ to $\sim 120 \mathrm{kHz}$ by choosing different sets of apertures and subharmonics of the ring frequency ( $\mathrm{MHz}$ range). Also the opening time of the system can be varied from some nanoseconds to several microseconds to meet the needs of a broad range of applications. Adjusting these parameters, the device can be used either for the generation of single light pulses or pulse packages from a microwave driven, continuous He gas discharge lamp or from storage rings which are otherwise often considered as quasi-continuous light sources. This chopper can be utilized for many different kinds of experiments enabling, for example, unambiguous time-of-flight (TOF) multi-electron coincidence studies of atoms and molecules excited by a single light pulse as well as time-resolved visible laser pump x-ray probe electron spectroscopy of condensed matter in the valence and core level region. (C) 2012 American Institute of Physics. [doi:10.1063/1.3677329]
\end{abstract}

\section{INTRODUCTION}

Today's synchrotron radiation facilities provide light pulses at typical repetition rates of several 100's $\mathrm{MHz}$ as given by the number of electron bunches orbiting in the machine, the circumference of the storage ring and the near speed of light of the circulating particles. Since the total number of charges in one bunch is limited, multi-bunch filling patterns are commonly used to achieve a suitably high ring current and, accordingly, high light intensity as required by many users' experiments for which the time structure of the synchrotron does not matter.

For experiments for which the time structure is of importance, storage rings can be operated in different filling patterns as, for instance, in hybrid mode, where one electron bunch (the "hybrid bunch") is well separated in time from the adjacent ones, or in pure single bunch mode. The latter decreases the repetition rate of the $\mathrm{x}$-ray pulses by two orders of magnitude. As an example, this operation mode is frequently used at the BESSY-II facility in Berlin where the repetition rate of the light is then reduced from $500 \mathrm{MHz}$ to about 1.25 $\mathrm{MHz}$. Some timing experiments in the VUV and x-ray spectral region require even lower light pulse rates in the order of $10-100 \mathrm{kHz}$. To meet these needs, a mechanical chopper system can be used to block a certain fraction of the light pulses.

Different chopper systems for single light pulse extraction at synchrotron radiation facilities have been developed during the years. The range of technical solutions comprises

\footnotetext{
a) Electronic mail: stefan.plogmaker@fysik.uu.se.

b)Electronic mail: hakan.rensmo@fysik.uu.se.

c)Electronic mail: raimund.feifel@fysik.uu.se.
}

rotating crystals or mirrors, triangular-shaped metal plates, modified rotors of turbo molecular pumps as well as the classical chopper design of a rotating disc with its rotational axis parallel to the propagation direction of the light beam (see Refs. 1-11, and references therein).

Rotating crystals and triangular-shaped metal plates enable very short opening times, but run into limitations with respect to the repetition rates practically achievable. For instance, using a rotational frequency of $1 \mathrm{kHz}$, the triangledesign reaches a throughput of up to $3 \mathrm{kHz}$ if synchronized properly with the radio frequency signal of the storage ring. Pulse selectors based on rotating crystals have not reached this frequency yet.

Another interesting technical solution is the so-called "hamster wheel" chopper built on a turbo molecular pump. ${ }^{10}$ This has the advantages of being comparatively cheap, easily vacuum compatible and reliable at high motor speed. In such a design, a cylinder with a certain number of apertures is mounted on top of the rotor of the turbo pump with its rotational axis oriented perpendicular to the synchrotron beam. That is, the light passes through two apertures on opposing sides of such a hamster wheel. Multiple apertures in the cylinder enable much higher light pulse frequencies compared to the rotating triangle or crystal solutions, but the only device of this kind reported hitherto ${ }^{10}$ is not synchronized with the storage ring. Furthermore, slits on opposing sides of the wheel cannot both be at the focus of a convergent light beam.

In this work, we report on a novel chopper system which is based on the classical spinning disc solution oriented with its rotational axis along the propagation direction of the light beam and which is synchronized to the radio frequency of the electron storage ring. The mechanical design of this de- 
vice allows for simple adjustment or exchange of the chopper discs and hence allows one to obtain a broad range of opening times and repetition rates of the light pulses transmitted (few kHz-120 kHz). Two examples are given to demonstrate the versatility of this chopper system. The first one is a showcase for how the device reduces substantially the light pulse frequency at a synchrotron radiation facility to a rate which suits the actual experimental needs, and the second one demonstrates how the continuous intensity pattern of a homelaboratory, microwave driven $\mathrm{He}$ gas discharge lamp as well as the pseudo-continuous intensity pattern of an electron storage ring operated in multi-bunch mode can be modulated with this chopper for time-resolved pump-probe experiments.

\section{CHOPPER DESIGN}

\section{A. Timing considerations of the chopper system for single pulse selection}

When operating in single bunch mode, an electron storage ring has a typical bunch separation time of the order of several hundreds of nanoseconds (ns), as for example, in the case of BESSY-II $\sim 800 \mathrm{~ns}$ (exactly $800.5515 \mathrm{~ns}$ ), and a pulse length of some tens to hundreds of picoseconds (ps) (BESSY-II: 30 ps). To extract single pulses from BESSY-II, the full opening time of the chopper system has to be somewhat shorter than one full ring period in order to prevent two pulses or parts of such passing through. Furthermore, with respect to a specific application of the chopper device discussed here, namely, multi-electron coincidence experiments using a several meter long magnetic bottle spectrometer, the desired minimum separation time of the light pulses is $\sim 10 \mu \mathrm{s}$ or more, which determines, in this case, the maximum number of openings on a disc rotating at a given fixed frequency.

In order to ensure the highest possible photon flux for the experiment, the beam chopper should preferably be synchronized with the radio frequency of the storage ring. Without synchronization the transmitted pulses would come at random intervals and; hence, the intensity per pulse would fluctuate strongly, leading to a substantial reduction in the overall transmitted intensity. Only a synchronized chopper system will supply the experiment with a stable light intensity and transmit the pulses at a constant frequency.

\section{B. Main design considerations}

After having studied different design principles, such as rotating cylinders, triangles, and mirrors, we decided to explore the classical chopper design of a spinning disc with the rotational axis parallel to the propagation direction of the light beam. As it turned out, this has practical advantages in terms of an easy alignment of the system, and it allows for much higher repetition rates compared to alternative solutions based on rotating triangles, mirrors, or crystals.

Figures 1(a) and 1(b) give detailed insights into our chopper device. As can be seen, a set of metal discs, mounted onto the axis of an electric motor (details given in Sec. II C), comprises many slits, and the whole unit is housed in two standard DN100CF flanges, which serve both as supporting vacuum chamber and as a safety cover in case of an undesired event of disc failure. One important component to be noticed is a fixed slit mounted, for several purposes, on the backside of the disc arrangement. It blocks diffusively scattered x-ray radiation, and it can serve as a beam aperture as well as a differential pumping aperture. Furthermore, it facilitates the alignment of the whole system relative to the focus of the synchrotron radiation beam. A similar aperture is mounted on the diametrically opposite side of the system to be used in combination with a setup of a laser diode and detector for time referencing purposes.

\section{Motor and electronics}

In order to keep the costs as low as possible while aiming at a highly reliable system, a standard industrial brushless dc motor of Maxon EC 25 type (cf. Ref. 12) is used. Such a motor costs a fraction of a magnetic bearing system is comparatively small and easy to handle. To avoid the additional space for rotational feedthroughs the motor was equipped with vacuumcompatible ball-bearings. A customized motor control system from Peak Servo Corp./Eltrol, USA (cf. Ref. 13) in combination with a homemade, adjustable frequency divider was used to control the speed of the motor and to synchronize its rotation in both frequency and phase relative to the radio frequency bunch marker of the storage ring. In the frequency divider, a PIC microcontroller converts a potentiome-

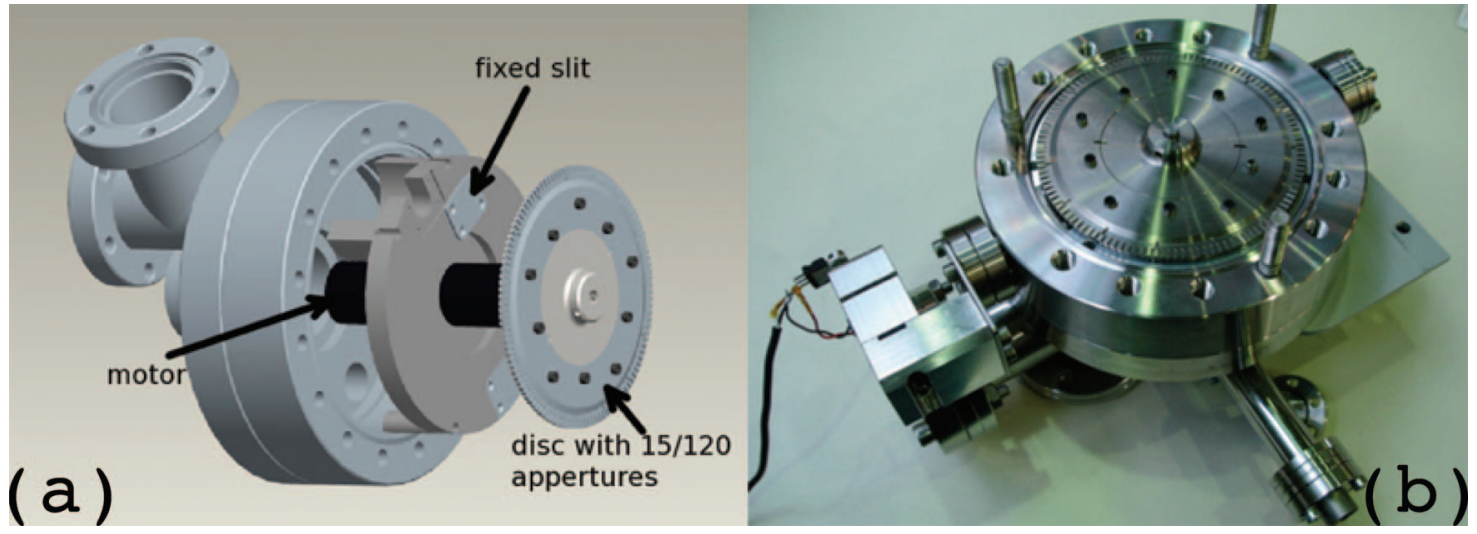

FIG. 1. (Color online) (a) Explosion drawing of the chopper system illustrating its main components; (b) a photograph of the manufactured system before mounting onto the vacuum system. 
ter setting to a digital equivalent, which is sent to a display and to a transistor-transistor logic (TTL) divide-by-n counter. The phase of the output frequency is set by a potentiometercontrolled TTL delay, adjustable over a range of several input frequency periods.

\section{Disc assembly}

To begin with, the design of the chopper discs followed two practical considerations. They had to be machinable in house, aiming for the best possible balance and accuracy in relative positioning of the apertures. They had also to be mountable directly onto the motor axis to avoid unnecessary space and costs for additional bearings and axes.

In the case of the BESSY-II experiments mentioned above, the required full opening time of $\sim 750 \mathrm{~ns}$ at a rotational frequency of about $650 \mathrm{~Hz}$ and a disc diameter of $100 \mathrm{~mm}$ resulted in a maximum aperture size $s_{\text {aperture }}$ of $\sim 76$ $\mu \mathrm{m}$ according to

$$
\frac{\tau \mathbf{d} \pi \mathbf{f}_{\text {rot }}}{2}=\mathbf{S}_{\text {aperture }}
$$

Here $\tau$ denotes the required full opening time, $d$ is the disc diameter and $f_{\text {rot }}$ is the rotational frequency of the disc. The factor of $1 / 2$ in this equation comes from the fact that the full opening time has to be used to calculate the maximum slit width. In this case the combination of one fixed slit of 76 $\mu \mathrm{m}$ in width and another $76 \mu \mathrm{m}$ wide slit on the disc results in an opening time of about $750 \mathrm{~ns}$ at $650 \mathrm{~Hz}$. In practice, it is more useful to divide this combination into a fixed slit of about $40 \mu \mathrm{m}$ in width and an aperture size of $110 \mu \mathrm{m}$ on the disc. This arrangement can compensate for some jitter in the phase lock and some small misalignment of the apertures on the disc relative to the light beam while still transmitting pulses with equal intensities.

The chopper disc assembly actually comprises two $\mathrm{Ti}$ discs mounted back to back. The two discs are equipped with exactly the same sets of apertures. The first set, located on the periphery of the discs, consists of 120 slits, and the second set, located on a circle somewhat further in the discs, of 15 slits. By rotating the two discs relative to each other, the effective slit width and hence the opening time of the chopper can be adjusted. We note that this part of the design makes it possible to manufacture the apertures with standard machines, resulting in a highly accurate relative positioning and shape of the slits.

Let us discuss briefly the usefulness of this design choice on the grounds of actual experimental needs. In the case of pure single pulse extraction as outlined above for BESSY-II experiments, the opening time is preferably adjusted to be slightly shorter than the time for one full ring cycle. In this way, one obtains for the experiment the highest possible photon flux at the desired repetition rate without undesired double pulse structures, taking the mechanical jitter of the device into account.

If the chopper system is used instead to pulse continuous light sources such as a microwave driven He gas discharge lamp or pseudo-continuous light sources, such as a storage ring operated in multi-bunch mode, this design principle can
TABLE I. X-ray intensities and transmitted frequencies recorded for different operation conditions of the chopper system.

\begin{tabular}{lccc}
\hline \hline & Fixed slit & 15 slit circle & 120 slit circle \\
\hline $\begin{array}{l}\text { Applied integer divider } \\
\begin{array}{l}\text { Theoretical transmitted } \\
\text { frequency }[\mathrm{kHz}]\end{array}\end{array}$ & 1249.138 & 128 & 16 \\
$\begin{array}{l}\text { Measured transmitted } \\
\text { frequency } f_{\text {trans }}[\mathrm{kHz}]\end{array}$ & 1249.138 & 9.747 & 78.07 \\
$\begin{array}{l}\text { Phase lock stability [chosen } \\
\text { frequency/ftrans }\end{array}$ & $\ldots$ & 0.9988 & 0.9999 \\
$\begin{array}{l}\text { Transmitted intensity } \\
\text { Intensity without chopper/ }\end{array}$ & $15 \mathrm{nA}$ & $0.12 \mathrm{nA}$ & $0.95 \mathrm{nA}$ \\
transmitted intensity & 1 & 125 & 15.8 \\
\hline \hline
\end{tabular}

be utilized to adjust the opening time of the device according to the lifetime of the electronic states studied in a specific sample. For instance, electronic states of microsecond lifetimes do not necessarily have to be measured with short picosecond or nanosecond pulses, but can be studied instead with microsecond pulses (or microsecond pulse packages) to increase the flux and statistics in the cases of photon-hungry experiments.

Furthermore, with respect to synchrotron radiation facilities operated in hybrid bunch mode, we can foresee that this design can be utilized to extract solely the hybrid bunch while blocking the remaining multi-bunch part of this filling pattern. This will open up the possibility to carry out pure singlebunch experiments, while other experiments can still make use simultaneously of the full multi-bunch character of the storage ring.

\section{PROOF OF PRINCIPLE OF THE SYNCHRONIZED CHOPPER SYSTEM}

The proof of principle of the synchronization of this chopper system with an electron storage ring was established by monitoring the transmitted x-ray pulses with a multichannel plate (MCP) detector. Measurements of both the synchrotron radiation intensity using a picoammeter and the actual time structure of the light pulses using a digital oscilloscope were made at beam line U49/2-PGM-2 (cf. Ref. 14) of the BESSY-II facility and are summarized in Table I. The results of the pulse structure measurements are also shown in Fig. 2 for the two different sets of apertures mentioned above. As can be seen, stable periodic signals of about $9.747 \mathrm{kHz}$ and $78.06 \mathrm{kHz}$, respectively, were found, which imply that exactly one pulse per aperture opening passes through and that the alignment of the slits as well as the jitter of the phase lock fulfill well the requirements on the desired timing accuracy.

\section{EXPERIMENTS AND RESULTS}

The new chopper system has been used for different kinds of applications such as multi-electron coincidence experiments on atoms and molecules based on a long magnetic bottle spectrometer (see, e.g., Refs. 15-17, and references therein) with nanosecond timing resolution, and for visible laser pump x-ray probe electron spectroscopy studies of con- 


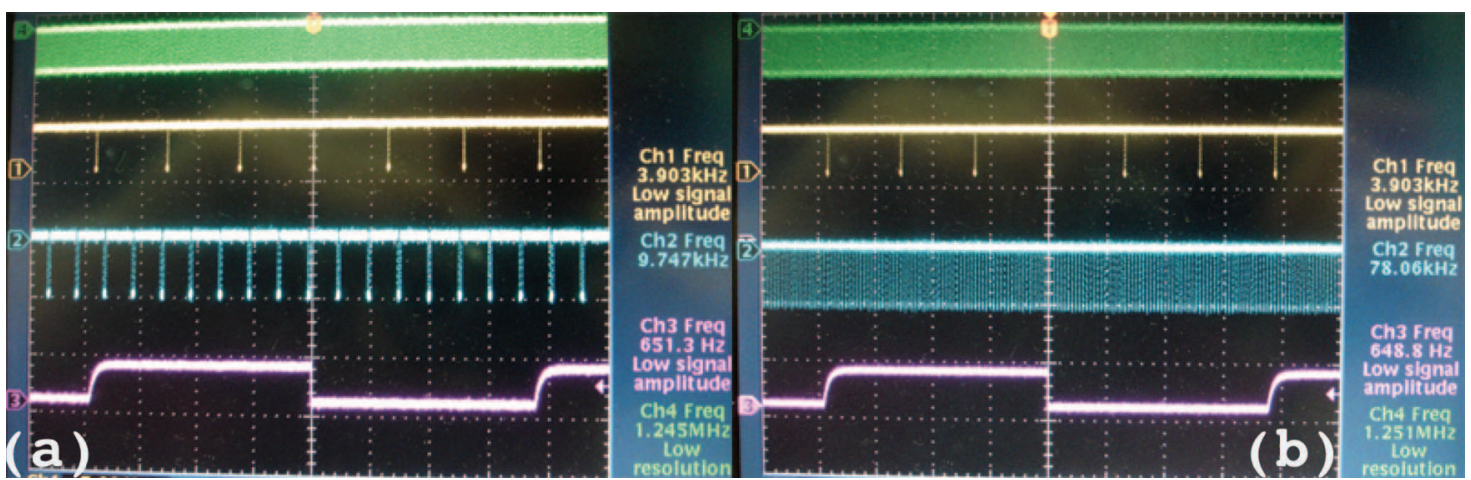

FIG. 2. (Color online) The radio frequency signal of the BESSY-II ring (green), the frequency input of the motor driver (yellow), the MCP light pulse signal (blue), and the motor hall sensor (red) monitored on a digital oscilloscope for (a) a set of 15 apertures and (b) a set of 120 apertures.

densed matter in the microsecond time region. These experiments will be described and discussed in more details in what follows.

\section{A. Adjusted repetition rate of synchrotron light pulses for multi-electron coincidence studies}

Single bunch operation at BESSY-II with a pulse length of $30 \mathrm{ps}$ and a repetition rate of $1.25 \mathrm{MHz}$ provides the possibility for fast timing measurements. This is of great interest for multi-electron coincidence studies of photoionized atoms and molecules based on a highly efficient and long magnetic bottle time-of-flight spectrometer (cf. Refs. 15-17). As illustrated schematically in Fig. 3, in such an experiment the flight times of the electrons, created at the interaction point of light and matter, to a several meter distant detector are measured and used to establish their kinetic energies. In particular electrons with near zero kinetic energy can cause timing problems if their flight times exceed the separation time of two consecutive ionizing light pulses. By applying a small electrical dc field to the interaction region, one can ensure that even electrons with initial zero kinetic energy reach the $\sim 2.2 \mathrm{~m}$ distant detector in less than $10 \mu$ s while preserving the resolv-

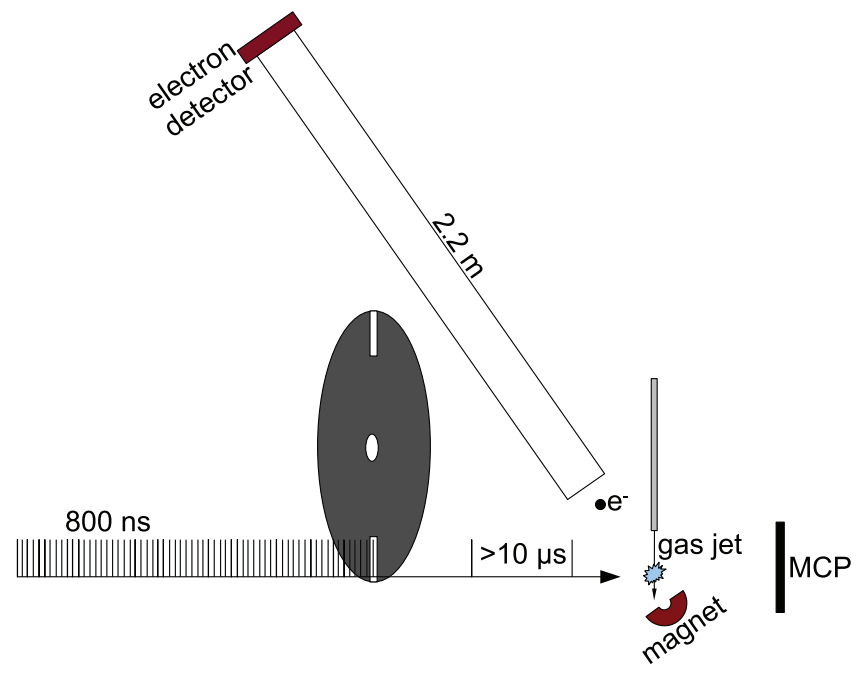

FIG. 3. (Color online) Schematic illustration of the new chopper system combined with a $2.2 \mathrm{~m}$ long multi-electron time-of-flight spectrometer based on a magnetic bottle (see Ref. 17, and references therein). The light pulse spacing is increased from about $800 \mathrm{~ns}$ to $>10 \mu \mathrm{s}$. ing power of the spectrometer. This comparatively long flight time range exceeds the typical inter-pulse spacing of the storage ring by a factor of $\sim 10$ or more and leads to overlapping spectral features originating from different ionization events.

One solution to this problem is to adjust the repetition rate of the radiation source with a chopper system as discussed here, blocking a certain fraction of the light pulses and thereby extending the time window between two subsequently transmitted pulses to be longer than $10 \mu$ s (see Fig. 3).

The new chopper system has been used for this purpose at beam line U49/2-PGM-2 (cf. Ref. 14) of the BESSY-II storage ring, and a typical result is shown in the upper panel of Fig. 4 which presents the single-photon excited valence triple ionization electron spectrum of $\mathrm{Kr}$ excited by $90 \mathrm{eV}$ photons. At this photon energy, one cannot establish unambiguously the flight times of electrons associated with a triple ionization event relative to the correct light pulse without a chopper, since in this case light pulse identification would require at

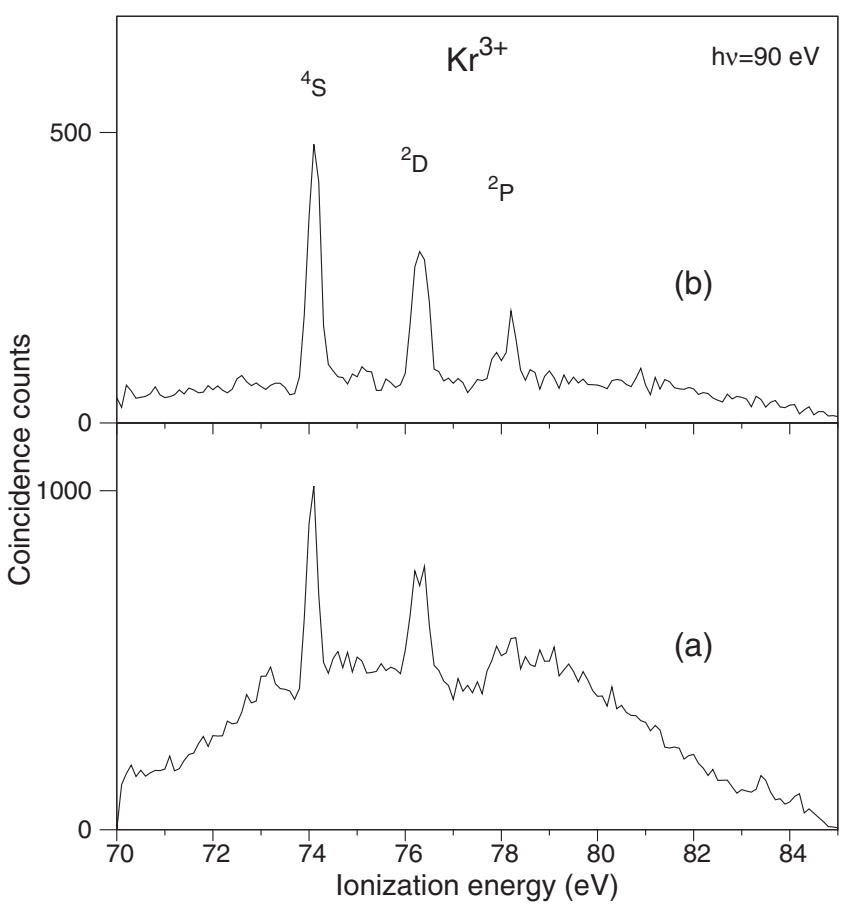

FIG. 4. Triple ionization electron spectra of Krypton recorded at $90 \mathrm{eV}$ photon energy without (a) and with (b) the chopper. See text for explanations of the differences. 
least one of the three electrons to have an energy of at least $\sim 21 \mathrm{eV}$ in order to arrive at the detector within one ring cycle.

The true electron flight times for the energy region shown can be recovered in analysis by trial and error, using the known triple ionization energies of $\mathrm{Kr}$ (see, e.g., Ref. 18) and the principle of energy conservation, but the necessary shift of the data unavoidably introduces a substantially enhanced background in the spectrum leading to the blurring of spectral features of interest as shown in the lower part of Fig. 4. The absence of an unambiguous time reference also makes photoionization of the $\mathrm{Kr} 3 d$ shell by 2 nd order light indistinguishable from photoionization by $90 \mathrm{eV}$ photons. As the cross section for triple ionization by cascade Auger decay from a $\mathrm{Kr} 3 d$-hole ${ }^{19,20}$ is much higher than direct triple ionization this process greatly interferes in the coincidence data, even though the amount of 2 nd order light is only a few percent of the 1st order light. In contrast, by establishing absolute electron flight times using the chopper, spectral contributions corresponding to photoionization of the $\mathrm{Kr} 3 d$ shell by 2 nd order light could be removed from the data shown in the upper panel of Fig. 4. This leads to a major reduction of background, revealing, e.g., the structure associated with the ${ }^{2} \mathrm{P}$ term.

\section{B. Laser pump x-ray probe experiments on charge transfer processes}

Time resolved pump-probe techniques can give important insights into, for example, the temporal evolution of excited states or charge transfer processes of atoms and molecules. Commonly, laser sources are used both for the pump and the probe pulses, which facilitate such measurements. Standard techniques detect transient absorption or emission of visible light, which limits the use, within the field of condensed matter, to the study of three-dimensional bulk properties. Furthermore, these methods give limited element specific information which, in contrast, can be obtained easily from x-ray and VUV-based measurements on core or localized valence levels.

To provide the possibility for pump-probe experiments utilizing the VUV radiation of a continuous wave homelaboratory He gas discharge lamp or the x-ray radiation of a storage ring operated in multi-bunch mode, such as the MAXII facility in Lund, the new chopper system has been used to generate microsecond $(\mu \mathrm{s})$ light pulses or pulse packages. The schematic layout for such experiments at a storage ring is illustrated in Fig. 5. In this case, the chopper system runs asynchronously and generates $\mu$ s x-ray pulse packages at a repetition rate of several $\mathrm{kHz}$. This in combination with a visible, nanosecond q-switch laser system can be used to perform visible pump VUV or X-ray probe experiments in the $\mu$ s time domain. No gating of the electron detector is necessary and hence a highly resolving hemispherical electrostatic energy analyzer can be used without modifications.

In order to exploit this technique, a pump-probe experiment on a dye-sensitized solar cell sample was carried out. In such a solar cell a semiconductor electrode, in this case $\mathrm{TiO}_{2}$, is sensitized with a light absorbing molecule. Under visible light illumination, the molecules adsorbed on the surface are expected to absorb photons and inject electrons into the $\mathrm{TiO}_{2}$.

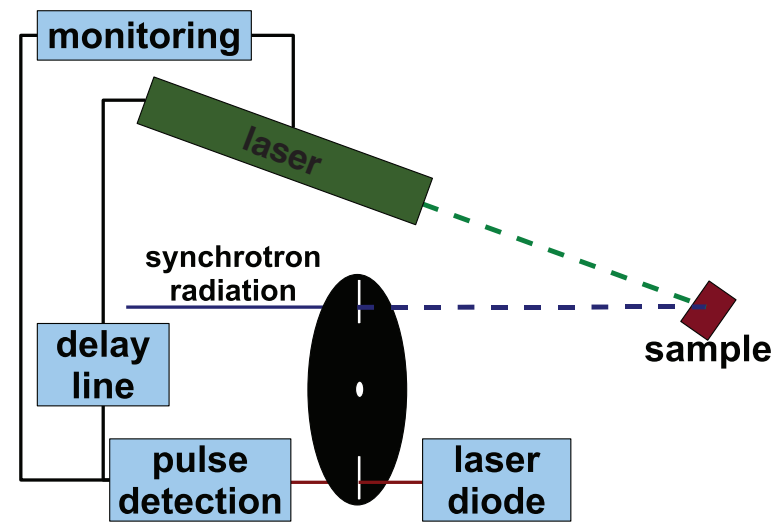

FIG. 5. (Color online) Schematic illustration of the setup for visible laser pump X-ray probe experiments as carried out at the MAX-II storage ring. The pump laser is triggered by a light signal available at the aperture opening and detected by optical means. The delay between the visible laser pulse and the $\mathrm{x}$-ray pulse is monitored on an oscilloscope.

A nanoporous $\mathrm{TiO}_{2}$ electrode sensitized with the ruthenium based dye-molecule 520DN (Solaronix) was chosen for the present study.

To optimize the experiment, the chopper system was first tested in the laboratory with a microwave driven He gas discharge lamp, and later on installed at beam line I 411 (cf. Ref. 21) of the MAX-II storage ring. It modulated the otherwise continuous intensity of the He lamp as well as the quasi-continuous intensity pattern of the synchrotron radiation at a repetition rate of $5 \mathrm{kHz}$ and with pulses of about $1 \mu \mathrm{s}$ in width (FWHM). A $532 \mathrm{~nm}$ q-switched laser system with $8 \mu \mathrm{J}$ per pulse was used as the visible pump and overlapped on the sample, both in time and space, with the synchrotron radiation. Valence band electron spectra, and, in particular in the case of the synchrotron radiation experiments, core level electron spectra were recorded in sequences of $5 \mathrm{~s}$, with and without laser illumination as well with different delay times between the laser pulse and the synchrotron radiation, and the results of some of the valence band measurements are shown in Fig. 6. As can be seen, the two spectra show a strong shift between the HOMO of the illuminated and non-illuminated case where the illuminated one shifts towards lower binding energy. In addition to these shifts in the valence band structure, we also observed similar shifts in the corresponding $\mathrm{C} 1 \mathrm{~s}$ core-level spectra. The amount of the shift depends strongly on the delay between the two pulses and is comparable with traditional transient absorption spectroscopy data. The experiments with the He gas discharge lamp as a VUV source (not shown here) showed similar behaviour of the samples.

The strong time-dependent shift of the spectra shows that pump probe measurements in the low microsecond range are feasible as a tool to investigate kinetics of, for example, dyesensitized solar cell samples or other light absorbing materials. To investigate the physics of such processes in detail, series of data with different delay times and photon energies need to be recorded and analyzed. 


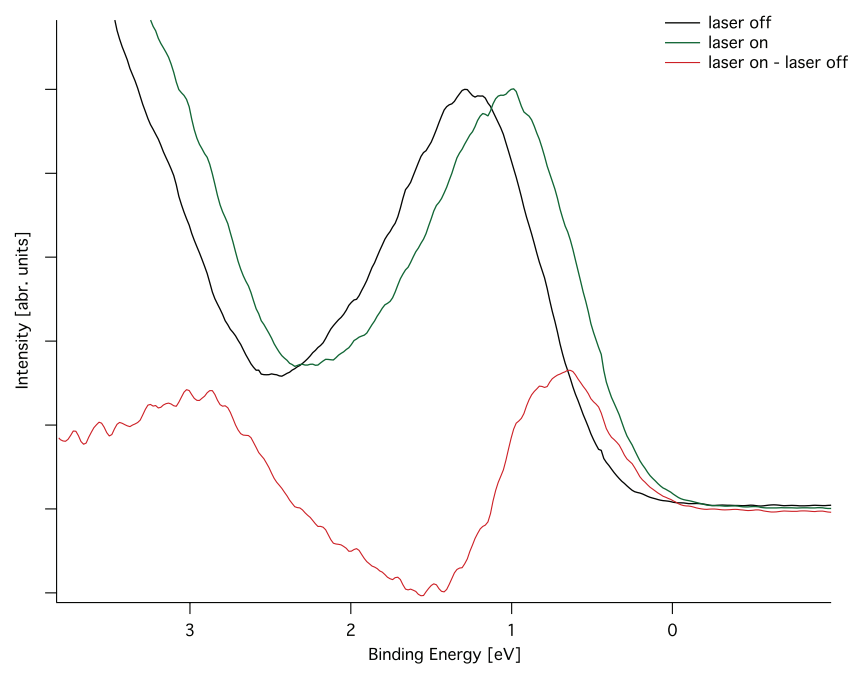

FIG. 6. (Color online) Valence band electron spectra measured at MAX-II using a photon energy of $60 \mathrm{eV}$ with and without laser illumination. The difference of the two spectra is also included.

\section{SUMMARY AND OUTLOOK}

The design of a new mechanical chopper system was presented and discussed. This system is highly versatile and can be synchronized with the time structure of a synchrotron radiation storage ring. The system is designed to operate at up to about $1000 \mathrm{~Hz}$ rotational frequency and thereby transmits light pulses at a frequency of up to about $120 \mathrm{kHz}$, depending on the number of apertures chosen on the spinning discs. Also the aperture size of this device can be adjusted easily, serving different kinds of experiments with a broad range of light pulse frequencies.

Two different kinds of applications were discussed. In the first kind of experiments, the repetition rate of the BESSY-II storage ring, operated in single-bunch mode, was successfully reduced from $1.25 \mathrm{MHz}$ to about $78 \mathrm{kHz}$, which suits much better multi-electron coincidence experiments based on a long time-of-flight magnetic bottle spectrometer as demonstrated for the case study of valence triple ionization of Krypton.

In another kind of experiment, the chopper system was used to modulate a continuous wave $\mathrm{He}$ gas discharge lamp as well as the MAX-II storage ring operated in multi-bunch mode, enabling visible laser pump VUV/x-ray probe experiments on the injection time of dye-sensitized solar cell materials. For the feasibility studies presented, the chopper device was adjusted to a repetition rate of $5 \mathrm{kHz}$ to match the specifications of the q-switch laser system employed, and the length of the VUV/x-ray pulses was set to about one $\mu$ s (FWHM).

Finally, the use of this chopper system can be foreseen to extract the main pulse from storage rings operated in hybrid bunch mode. For this kind of application, the opening time of the chopper would need to be adjusted to transmit only the photons originating from the isolated bunch and to block the remaining part of the multi-bunch structure. In this way, one could perform fast timing experiments akin to the ones which are done today in single-bunch mode, while other experiments could still utilize simultaneously the high photon flux provided by the complete multi-bunch filling pattern.

\section{ACKNOWLEDGMENTS}

This work has been financially supported by the Swedish Research Council (VR), the Göran Gustafsson Foundation (UU/KTH), and the Knut and Alice Wallenberg Foundation, Sweden. We are grateful to the support by the staff and colleagues at MAX-lab, Lund, and at BESSY-II, Berlin. This work was also supported by the European Community - Research Infrastructure Action under the FP6 "Structuring the European Research Area" Programm (through the Integrated Infrastructure Initiative "Integrating Activity on Synchrotron and Free Electron Laser Science" - Contract R II 3-CT-2004506008).

${ }^{1}$ A. D. Legrand, W. Schildkamp, and B. Blank, Nucl. Instrum. Methods A 275, 442 (1989)

${ }^{2}$ R. Tucoulou, D. V. Roshchupkin, O. Mathon, I. A. Schelokov, M. Brunel, E. Ziegler, and C. Morawe, J. Synchrotron Radiat. 5, 1357 (1998).

${ }^{3}$ D. Kosciesza and H. D. Bartunik, J. Synchrotron Radiat. 6, 947 (1999).

${ }^{4}$ A. McPherson, J. Wang, P. L. Lee, and D. M. Mills, J. Synchrotron Radiat. 7, 1 (2000).

${ }^{5}$ A. McPherson, W. K. Lee, and D. M. Mills, Rev. Sci. Instrum. 73, 2852 (2002).

${ }^{6}$ M. Gembicky, D. Oss, R. Fuchs, and P. Coppens, J. Synchrotron Radiat. 12, 665 (2005).

${ }^{7}$ M. Gembicky and P. Coppens, Synchrotron Radiat. 14, 1 (2006).

${ }^{8}$ A. Meents, B. Reime, M. Kaiser, X.-Y. Wang, R. Abela, E. Weckert, and C. Schulze-Briese, J. Appl. Crystallogr. 42, 901 (2009).

${ }^{9}$ M. Cammarata, L. Eybert, F. Ewald, W. Reichenbach, M. Wulff, P. Anfinrud, F. Schotte, A. Plech, Q. Kong, M. Lorenc, B. Lindenau, J. Raebiger, and S. Polachowski, Rev. Sci. Instrum. 80, 015101 (2009).

${ }^{10}$ K. Ito, F. Penent, Y. Hikosaka, E. Shigemasa, I. H. Suzuki, J. H. D. Eland, and P. Lablanquie, Rev. Sci. Instrum. 12, 123101 (2009).

${ }^{11}$ S. L. G. Husheer, J. M. Cole, T. d'Almeida, and S. J. Teat, Rev. Sci. Instrum. 81, 043905 (2010).

${ }^{12} \mathrm{See}$ http://www.maxonmotor.co.uk/ for detailed information.

${ }^{13}$ See http://www.peakservo.com/ for detailed information.

${ }^{14}$ D. R. Batchelor, R. Follath, and D. Schmeisser, Nucl. Instrum. Methods A 467, 470 (2001).

${ }^{15}$ J. H. D. Eland, O. Vieuxmaire, T. Kinugawa, P. Lablanquie, R. I. Hall, and F. Penent, Phys. Rev. Lett. 90, 053003 (2003).

${ }^{16}$ F. Penent, J. Palaudoux, P. Lablanquie, L. Andric, R. Feifel, and J. H. D. Eland, Phys. Rev. Lett. 95, 083002 (2005).

${ }^{17}$ J. H. D. Eland and R. Feifel, Chem. Phys. 327, 85 (2006).

${ }^{18}$ J. H. D. Eland, P. Linusson, L. Hedin, E. Andersson, J.-E. Rubensson, and R. Feifel, Phys. Rev. A 78, 063423 (2008).

${ }^{19}$ E. Andersson, S. Fritzsche, P. Linusson, L. Hedin, J. H. D. Eland, J.-E. Rubensson, L. Karlsson, and R. Feifel, Phys. Rev. A 82, 043418 (2010).

${ }^{20}$ J. Palaudoux, P. Lablanquie, L. Andric, K. Ito, E. Shigemasa, J. H. D. Eland, V. Jonauskas, S. Kucas, R. Karazija, and F. Penent, Phys. Rev. A 82, 043419 (2010).

${ }^{21}$ M. Bässler, A. Ausmees, M. Jurvansuu, R. Feifel, J.-O. Forsell, P. de Tarso Fonseca, A. Kivimäki, S. Sundin, S. L. Sorensen, R. Nyholm, O. Björneholm, S. Aksela, and S. Svensson, Nucl. Instrum. Methods A 469, 382 (2001). 